\title{
PERSONALIDAD Y USO DE LA COMPUTADORA EN ESTUDIANTES DE INGENIERÍA DE SISTEMAS
}

\section{Personality and use of computer in computing engineering students}

\author{
Walter Lizandro Arias Gallegos* \\ y María Alejandra Masías Salinas** \\ Universidad Católica San Pablo, Arequipa, Perú
}

\begin{abstract}
Resumen
En este estudio se analizan las relaciones entre la personalidad y el tiempo de uso de la computadora en estudiantes universitarios de la especialidad de ingeniería de sistemas. Se trabajó con una muestra de 67 estudiantes de una universidad privada de la ciudad de Arequipa a quienes se les aplicó una breve encuesta ad hoc y el Inventario de Personalidad de Eysenck. Los resultados muestran que el $44.7 \%$ de los estudiantes son de tipo de personalidad sanguíneo y que la mayoría de ellos le dedica más tiempo al uso de la computadora con fines de diversión, sin embargo, no se encontraron relaciones entre el uso de la computadora y la personalidad.
\end{abstract}

Palabras clave: Personalidad, computadora, ingeniería de sistemas.

\begin{abstract}
In the present study we analyze relations among personality and the computer time of using among university students of computing engineering. We work with a sample of 67 students from a private university located in Arequipa City, who fill a brief survey and the Eysenck Personality Inventory. The results show $44.7 \%$ of students are sanguine personality type the most of students dedicate more time using computers to get fun. Although, we did not find relations between the use of computer and personality.
\end{abstract}

Key words: Personality, computer, computing engineering.

\footnotetext{
* Psicólogo. Universidad Católica San Pablo, Arequipa, Perú. warias@ucsp.edu.pe

** Psicóloga. Universidad Católica San Pablo. Coordinadora del Grupo de Investigación Psyché.
} 


\section{INTRODUCCIÓN}

La personalidad, es una variable psicológica que ha recibido la atención de la academia psicológica a nivel global. Ello se aprecia en la cantidad de teorías que intentan explicar dicho constructo psicológico, y en las investigaciones que ha motivado la necesidad de comprender las implicancias psicosociales que tiene la personalidad en la educación, la salud, el trabajo y la vida social en general.

El Perú, no ha sido exento de la necesidad de profundizar el tema de la personalidad, siendo una de las primeras investigaciones de rigor académico, la realizada por el fundador de la psicología científica en el país: Walter Blumenfeld (1882-1967). La investigación de Blumenfeld implicó la aplicación del Inventario de Personalidad de Bernreuter en una muestra de jóvenes peruanos y norteamericanos, para valorar la introversión y la extroversión, determinando que los peruanos son menos extrovertidos que los norteamericanos y que los varones son más extrovertidos que las mujeres (Caycho, Arias y Barboza, 2015).

Una década más adelante, José Anicama (1974), realizó la estandarización del Inventario de Personalidad de Eysenck en la población de Lima. $\mathrm{Al}$ respecto, se debe mencionar, que en los países latinoamericanos, la teoría de Eysenck ha motivado diversos estudios (Mikusinski, Omar, Quintana \& Andrade, 1980; Gómez, 1985; Dergan, 1999; Montaña, Acosta \& Gerena, 2002, Schmidt, de Costa, Firpo, Vion \& Casella, 2008; Zambrano, 2011). Para el caso del Perú, aunque existen nuevos modelos teóricos que explican la personalidad, la teoría de Eysenck y sus instrumentos, son materia de constantes investigaciones y revisiones (Gómez, 1985; Arias, 2012; Dominguez, Villegas, Yauri, Aravena \& Ramírez, 2013; Arias, Infantes y Ceballos, 2016).

En ese sentido, modelos como la teoría de Millon (Fuentes, 2002) o el modelo de los Cinco Grandes (Ruíz, 2003), aunque son conocidos en el Perú, no han inspirado tantas investigaciones, como la teoría factorial de Eysenck. Podemos mencionar solo algunos estudios que responden a estas teorías, como la adaptación del Inventario de Estilos de Personalidad de Millon en una muestra de estudiantes universitarios peruanos (Sánchez, Thorne, Martínez, Niño de
Guzmán \& Argumero, 2002) o el estudio que relaciona la actitud hacia los residuos sólidos y los factores de personalidad según el modelo de Costa y McCrae (Machicao, 2012). Esta tendencia, se debe en parte, a que no hay un conocimiento profundo de nuevas propuestas teóricas sobre la personalidad, porque tienen más arraigo los modelos tradicionales como el de Eysenck o las teorías psicoanalíticas (Engler, 1999). La cuestión de fondo, es que hay se confunden las teorías de la personalidad con la psicología de la personalidad (Geldres y Arias, 2007). Además, las teorías más recientes de la personalidad se basan en procesamientos estadísticos que son desconocidos por la mayoría de psicólogos peruanos, como la regresión logística, las ecuaciones estructurales, el análisis factorial confirmatorio o la teoría de respuesta al ítem (Richaud, 2003).Este hecho dificultaría la comprensión de modelos teóricos como los Cinco Grandes Factores de la Personalidad; aunque por otro lado, este tipo de teorías, han sido criticadas por carecer de fundamento teórico (Richaud, 2002), a pesar de tener una sólida base estadística (Laak, 1996).

Encontraparte,el modeloteóricodeEysenck(1982) cuenta con bases biológico conductuales a partir de las investigaciones de Pavlov, de quien toma los conceptos de excitación e inhibición, muy relacionados con el arousal, aspecto clave en los estudios eysenckianos de la personalidad. Por otro lado, la investigación de la personalidad según el modelo de Eysenck, ha tocado temas muy variados, pero sobre todo dentro del marco de la salud y la psicología clínica: se ha reportado por ejemplo, que la dimensión de Psicoticismo de la teoría de Eysenck, es muy marcada etapas pre-mórbidas de la esquizofrenia (Lemos, 1989), y que altos puntajes en la dimensión de Neuroticismo se relacionan con ira, conductas antisociales y hostilidad (Montaña, Acosta y Gerena, 2002), así como con la intensidad emocional negativa, mientras que la Extraversión se relaciona con la intensidad emocional positiva (Chico, 2000).

Ahora bien, aunque son varios los estudios realizados sobre la base de la personalidad, y particularmente, tomando como fundamento la teoría de Eysenck, no son muchos los estudios que vinculen el uso de la tecnología con la personalidad. En el Perú, puntualmente, en Lima, existen diversos estudios sobre la psicología y la tecnología, pero están más avocados 
a las adicciones tecnológicas (Astonitas, 2005; Salas, 2014; Salas y Escurra, 2014; Escurra y Salas, 2014). En parte porque es un tema con implicancias clínicas relevantes, pero también, porque es un fenómeno que se está extendiendo con bastante rapidez. En Arequipa, el interés por la tecnología, está generando múltiples líneas de investigación sobre sus implicancias en la educación (Arias, 2015), el trabajo (Arias y Salinas, 2014), y las adicciones tecnológicas (Flores, Gamero, Arias, Melgar, Sota \& Ceballos, 2015), entre otros.

Sin embargo, un tema desatendido en nuestro medio, es el de la personalidad y la tecnología, a pesar de que la personalidad está presente en diversos usos que le damos a la tecnología, desde la creación del password (Infante, 2013), como en el uso de servicios de asistencia psicológica on line (Vallejo, Jordán, Díaz, Comeche, Ortega, Amor \& Zubeidat, 2008), la adicción a la Internet (de Gracia, Vigo, Fernández y Marcó, 2002) y el uso de redes sociales (Aguilar \& Said, 2010). En el estudio de Sanabria (2004) por ejemplo, se encontró que las personas introvertidas eran más proclives a consumir cibersexo, debido a que su timidez y escasa vida social, dificulta sus relaciones interpersonales con el sexo opuesto. El uso del Facebook en cambio suele ser más frecuente en las mujeres y las personas extrovertidas, dadas sus altas tendencias hacia la socialización, aunque también se ha visto que los introvertidos enriquecen su vida social a través de las redes sociales porque no es necesario el contacto personal y porque pueden construir una identidad virtual más aceptable socialmente, de como en realidad son (Escalante y Agrelo, 2012). La investigación de Astonitas (2005) concluyo que bajos niveles de la dimensión de responsabilidad, en un grupo de universitarios peruanos, se relacionaba con la adicción a la Internet, pero que los más excitables serían quienes presentan mayor riesgo, tal y como ocurre con la adicción a los videojuegos (Estalló, 1994).

El presente estudio de carácter exploratorio, pretende valorar las relaciones entre la personalidad y el uso de la computadora, en términos de tiempo dedicado y tipo de actividades realizadas; en un grupo de estudiantes universitarios de la carrera de ingeniería de sistemas. Se trata de un estudio cuantitativo y de alcance correlacional y comparativo, pues también se analizarán las diferencias en función del sexo de los participantes.

\section{MÉTODO}

\section{Muestra}

La muestra está constituida por 67 estudiantes del Programa Profesional de Ingeniería de Sistemas de una universidad privada de la ciudad de Arequipa. El 82\% (55) son varones y el $18 \%$ (12) mujeres con una edad promedio de 21 años y una desviación estándar de \pm 3.367 . Todos pertenecen a una condición económica correspondiente a un nivel socioeconómico medio y cursan el segundo año de estudios.

\section{Instrumentos}

Ficha de datos: recoge información relativa al sexo, la edad, las horas diarias dedicadas al uso de la computadora con fines laborales, de estudio, de diversión y las horas semanales de uso de la computadora.

Inventario de personalidad de Eysenck (EPI) para adultos forma B. Consta de 57 ítems de naturaleza dicotómica (Sí-No) que valora dos dimensiones de la personalidad: extroversión-introversión y estabilidadinestabilidad. De las combinaciones de estas dimensiones se obtienen cuatro tipos de personalidad: sanguíneo, colérico, flemático y melancólico. Es aplicable a personas de ambos sexos con una edad mínima de 18 años. Se utilizó la versión estandarizada por Anicama (1974) para el Perú.

\section{Procedimiento}

Todos los estudiantes fueron evaluados en sus respectivas aulas de estudio, con el permiso de los profesores correspondientes, previa autorización de las autoridades competentes. Se recogieron los datos en los turnos mañana, tarde y noche; con una duración aproximada de 20 minutos. La aplicación de los instrumentos fue colectiva y bajo la supervisión de los autores. Los datos fueron procesados mediante el programa SPSS 19.0 a través de pruebas estadísticas paramétricas, según el nivel de medición de las variables de estudio. 


\section{RESULTADOS}

En la Tabla 1 se aprecia que la media de horas de uso de la computadora dedicadas al trabajo es de 3.8 dentro de un rango de 0 a 10 horas y una desviación típica de 2, mientras que al estudio es de 3.9 (con un rango de 0.5 a 10 horas con una desviación típica de 2) y a la diversión, es de 4.2 (con una desviación estándar

Tabla 1

Estadísticos descriptivos

\begin{tabular}{lccrrrrr}
\hline & $\begin{array}{c}\text { Horas de } \\
\text { trabajo }\end{array}$ & $\begin{array}{c}\text { Horas de } \\
\text { estudio }\end{array}$ & $\begin{array}{c}\text { Horas de } \\
\text { diversión }\end{array}$ & $\begin{array}{c}\text { Horas por } \\
\text { semana }\end{array}$ & Neuroticismo & Extroversión Veracidad \\
\hline Media & 3.805 & 3.9 & 4.208 & 59.534 & 10.238 & 14.253 & 3.552 \\
Mediana & 4 & 4 & 2.5 & 35 & 10 & 15 & 4 \\
Moda & 5 & 4 & 1 & 4 & 5 & 15 & 4 \\
Desv. típ. & 2.039 & 2.078 & 3.886 & 18.472 & 4.479 & 2.808 & 1.417 \\
Varianza & 4.161 & 4.318 & 15.104 & 325.454 & 20.063 & 7.889 & 2.008 \\
Asimetría & 0.564 & 0.784 & 1.828 & 7.933 & 0.172 & -0.433 & 0.022 \\
Curtosis & 0.720 & 1.526 & 4.621 & 64.206 & -0.976 & -0.262 & -0.166 \\
Mínimo & 0 & 0.5 & 1 & 0.5 & 2 & 6 & 0 \\
Máximo & 10 & 10 & 20 & 150 & 19 & 19 & 7 \\
\hline
\end{tabular}

Con respecto a la personalidad, las puntuaciones en el dimensión de Neuroticismo, arrojan una media de 10.23 con una desviación típica de 4.4, mientras que Extroversión registra una media de 14.25 (y una desviación estándar de 2.8), y Veracidad 3.55 (con una desviación estándar de 1.4). Esto sugiere que los de 3.8 dentro de un rango de 1 a 20). Asimismo, el promedio de horas semanales de uso de la computadora es de 59.5 con una desviación de 18.4 y un rango de 0 a 150 horas. Esto supone que la mayor parte del tiempo que los estudiantes usan la computadora, está destinado a la diversión.

Tabla 2

Frecuencias y porcentajes de tipos de personalidad según Eysenck

\begin{tabular}{lccc}
\hline & Frecuencia & Porcentaje & Porcentaje acumulado \\
\hline Sanguíneo & 30 & 44.776 & 44.776 \\
Colérico & 12 & 17.910 & 62.686 \\
Melancólico & 12 & 17.910 & 80.597 \\
Flemático & 13 & 19.402 & 100 \\
Total & 67 & 100 & \\
\hline
\end{tabular}

Por otro lado, al hacer las correlaciones entre las puntuaciones directas de las dimensiones de personalidad con el tiempo dedicado al uso de la computadora y la edad, se tiene que a mayor edad, los estudiantes dedican más horas de estudio $(r=0.214)$, estudiantes tienen una personalidad con tendencia a la estabilidad y la extroversión, lo que se corrobora con el hecho de que el $44.7 \%$ de ellos, presenta una personalidad sanguínea, mientras que el $19.4 \%$ es flemático, el $17.9 \%$ es colérico y en igual porcentaje, son melancólicos (ver Tabla 2). mientras que los que dedican más horas al estudio, también lo hacen al trabajo $(\mathrm{r}=0.284)$. Asimismo, quienes dedican más horas al trabajo también le dedican más horas al uso de la computadora con fines de diversión $(r=0.262)$, pero la correlación fue más 
fuerte y significativa entre quienes le dedican tiempo al estudio y la diversión $(\mathrm{r}=0.436)$. También se observa que los estudiantes que le dedican más tiempo al trabajo, son quienes usan la computadora mayor cantidad de horas a la semana $(r=0.229)$ (ver Tabla $3)$.

Tabla 3

Correlaciones entre las variables cuantitativas

\begin{tabular}{|c|c|c|c|c|c|c|c|c|}
\hline & Edad & $\begin{array}{c}\text { Horas de } \\
\text { trabajo }\end{array}$ & $\begin{array}{c}\text { Horas de } \\
\text { estudio }\end{array}$ & $\begin{array}{l}\text { Horas de } \\
\text { diversión }\end{array}$ & $\begin{array}{c}\text { Horas por } \\
\text { semana }\end{array}$ & Neuroticismo & Extroversión & Veracidad \\
\hline$\overline{\text { Edad }}$ & 1 & 0.148 & $0.214^{*}$ & -0.134 & -0.081 & -0.045 & 0.132 & 0.012 \\
\hline H. de trabajo & & 1 & $0.284 *$ & $0.262 *$ & $0.229 *$ & -0.123 & -0.132 & -0.120 \\
\hline H. de estudio & & & 1 & $0.436 * *$ & 0.157 & -0.073 & 0.014 & $0.291 *$ \\
\hline H. de diversión & & & & 1 & 0.016 & 0.078 & -0.005 & -0.072 \\
\hline H. por semana & & & & & 1 & 0.133 & -0.115 & 0.101 \\
\hline Neuroticismo & & & & & & 1 & -0.053 & $-0.307 * *$ \\
\hline Extroversión & & & & & & & 1 & -0.035 \\
\hline Veracidad & & & & & & & & 1 \\
\hline
\end{tabular}

$* \mathrm{p}<0.05 ; * * \mathrm{p}<0.01$

Con respecto a la personalidad, solo se encontraron relaciones significativas entre las horas de uso de la computadora dedicadas al estudio y la veracidad $(\mathrm{r}=$ 0.291), lo que sugiere que quienes más estudian son los más veraces, mientras que el neuroticismo se relacionó negativa y significativamente con la veracidad ( $\mathrm{r}=$ 0.307 ), es decir que quienes dicen más la verdad son los menos inestables (Tabla 3).

Tabla 4.

Comparaciones según sexo con la prueba t Student

\begin{tabular}{|c|c|c|c|c|c|c|c|}
\hline & Sexo & $\mathbf{N}$ & Media & Desviación típ. & $\mathbf{t}$ & gl & p \\
\hline Horas de trabajo & $\begin{array}{l}\text { Varón } \\
\text { Mujer }\end{array}$ & $\begin{array}{r}42 \\
8\end{array}$ & $\begin{array}{l}3.648 \\
4.625\end{array}$ & $\begin{array}{l}2.184 \\
0.517\end{array}$ & -2.545 & 65 & 0.014 \\
\hline Horas de estudio & $\begin{array}{l}\text { Varón } \\
\text { Mujer }\end{array}$ & $\begin{array}{l}44 \\
11\end{array}$ & $\begin{array}{l}4.011 \\
3.454\end{array}$ & $\begin{array}{l}2.176 \\
1.634\end{array}$ & 0.940 & 65 & 0.358 \\
\hline Horas de diversión & $\begin{array}{l}\text { Varón } \\
\text { Mujer }\end{array}$ & $\begin{array}{r}39 \\
9\end{array}$ & $\begin{array}{l}4.692 \\
2.111\end{array}$ & $\begin{array}{l}4.098 \\
1.691\end{array}$ & 2.983 & 65 & 0.005 \\
\hline Horas de semana & $\begin{array}{l}\text { Varón } \\
\text { Mujer }\end{array}$ & $\begin{array}{l}55 \\
12\end{array}$ & $\begin{array}{l}67.304 \\
23.916\end{array}$ & $\begin{array}{r}198.351 \\
24.422\end{array}$ & 1.568 & 65 & 0.121 \\
\hline Neuroticismo & $\begin{array}{l}\text { Varón } \\
\text { Mujer }\end{array}$ & $\begin{array}{l}55 \\
12\end{array}$ & $\begin{array}{r}9.945 \\
11.583\end{array}$ & $\begin{array}{l}4.360 \\
4.962\end{array}$ & -1.057 & 65 & 0.307 \\
\hline Extroversión & $\begin{array}{l}\text { Varón } \\
\text { Mujer }\end{array}$ & $\begin{array}{l}55 \\
12\end{array}$ & $\begin{array}{r}14.309 \\
14\end{array}$ & $\begin{array}{l}2.761 \\
3.133\end{array}$ & 0.315 & 65 & 0.756 \\
\hline Veracidad & $\begin{array}{l}\text { Varón } \\
\text { Mujer }\end{array}$ & $\begin{array}{l}55 \\
12\end{array}$ & $\begin{array}{l}3.527 \\
3.666\end{array}$ & $\begin{array}{l}1.425 \\
1.435\end{array}$ & -0.305 & 65 & 0.764 \\
\hline
\end{tabular}


Un último análisis de la data, implicó hacer las comparaciones entre los valores de nuestras variables en función del sexo de los estudiantes, con la salvedad de que hubo un número significativamente mayor de estudiantes varones. Esto sugiere que los valores que a continuación reportamos, deben ser vistos con cautela. En base a la aplicación de la prueba t Student, se tiene que las mujeres dedican más tiempo al uso de la computadora con fines de trabajo $(\mathrm{p}<0.014)$, mientras que los varones le dedican más tiempo al uso de la computadora para divertirse $(\mathrm{p}<0.005)$.

\section{DISCUSIÓN}

Los resultados de nuestro estudio, indican que no hay relación entre la personalidad, según el modelo de Eysenck, y el tiempo dedicado al uso de la computadora, en estudiantes de ingeniería de sistemas. Estos resultados son similares a los reportados por Astonitas (2005) que aunque sí encontró que la dimensión de responsabilidad puede predecir la adicción a la Internet, no pudo concluirse que el tiempo dedicado a la computadora, sea un indicador de la adicción a la Internet. Otros estudios, han reportado que son los extrovertidos quienes son más propensos al juego patológico (Echeburúa, 1992), a la adicción a los videojuegos (Estalló, 1994) y a las redes sociales (Escalante y Agrelo, 2012).

Sin embargo, existen diversas explicaciones para la tendencia hacia el uso de la computadora, tanto en introvertidos como en extrovertidos. Los introvertidos, debido a su timidez, suelen preferir no interactuar con personas (Caycho, Castilla, Urrutia, Valdivia y Shimabukuro, 2013), por tanto serían más propensos al uso de tecnologías, pues estas reducen el contacto personal entre el emisor y el receptor. Por otro lado, los extrovertidos podrían usar más la computadora con fines de socialización a través de las redes sociales.

En nuestro estudio, aunque se ha encontrado que los estudiantes dedican más tiempo a usar la computadora para divertirse, sobre todo los varones, no podemos determinar qué tipo de diversión es la que prefieren, lo cual deja abierta un amplia posibilidad de usos, pero considerando nuestro contexto actual, podemos inferir que se trata de juegos en red y el uso de redes sociales. En ese sentido, la falta de especificación de los usos de la computadora, es una limitación que debe tomarse en cuenta. De la misma manera, la muestra y la procedencia de una sola universidad, limitan la generalización de los datos a otras universidades y a otras especialidades profesionales, que ni sean la ingeniería de sistemas.

Estudios posteriores, deben profundizar en este tema, y considerar las limitaciones de la presente investigación. Una dato valioso, empero, que hemos reportado, es el hecho de que los estudiantes no priorizan sus responsabilidades, sean estas laborales o académicas; pues utilizan más la computadora con la finalidad de despejarse y divertirse, que de estudiar o trabajar. Esto puede tener relación con los altos niveles de fracaso académico en la universidad peruana y con las deficientes capacidades profesionales que presentan algunos profesionales en nuestro país (Arias, 2013). Es necesario por tanto, que los estudiantes tomen conciencia de su rol como futuros profesionales y se comprometan más con sus estudios, priorizando el tiempo que le dedican al trabajo intelectual. Por otro lado, las autoridades universitarias, deben de velar por la exigencia académica de los estudiantes y por la correcta selección de los ingresantes a la universidad, porque realizar estudios universitarios, supone cierto grado de interés académico y una preocupación constante por la generación de conocimiento.

\section{REFERENCIAS}

Aguilar, D. E. \& Said, E. (2010). Identidad y subjetividad en las redes sociales virtuales: caso de Facebook. Zona Próxima, 12, 190-207.

Anicama, J. (1974). Rasgos básicos de la personalidad de la población de Lima. Un enfoque experimental. Estandarización del EPI-B (Tesis de bachiller). Lima: UNMSM.

Arias, W.L. (2012). Hans Jürgen Eysenck (1916-1997): El infatigable investigador de la personalidad. Revista de Psicología de la UCV, 14(1), 118-126.

Arias, W. L. (2013). Crisis de la universidad en el Perú: Un problema de su naturaleza y su identidad. Educación, 19, 23-39. 
Arias, W. L. (2015). Tecnologías de la información y la comunicación en colegios públicos y privados de Arequipa. Interacciones, 1(1), 11-28.

Arias, W. L. \& Masías, M. A. (2014). Ocio cibernético en trabajadores de instituciones públicas y privadas de Arequipa. Ciencia \& trabajo, 16(50), 88-92.

Arias, W. L.; Infantes, A. E. \& Ceballos, K. D. (2016). Personalidad, inteligencia emocional y estilos de afrontamiento en los conciliadores de la ciudad de Arequipa. Interacciones, 2(1), 21-32.

Astonitas, L. M. (2005). Personalidad, hábitos de consumo y riesgo de adicción al Internet en estudiantes universitarios. Revista de Psicología de la PUCP, 23(1), 65-112.

Caycho, T.; Arias, W. L. \& Barboza, M. (2015). Correspondencia entre Walter Blumenfeld $y$ Edwin G. Boring (1956-1958). Arequipa: Adrus Editores.

Caycho, T.; Castilla, H.; Urrutia, C.; Valdivia, A. \& Shimabukuro, M. (2013). Análisis psicométrico preliminar de la Escala de Timidez Revisada de Check y Buss en adolescentes y jóvenes peruanos. Psychologia: Avances de la Disciplina, 7(2), 1324.

Chico, E. (2000). Intensidad emocional y su relación con extraversión y neuroticismo. Psicothema, 12(4), 568-573.

De Gracia, M. G.; Vigo, M. V.; Fernández, M. J. \& Marcó, M. (2002). Problemas conductuales relacionados con el uso de Internet: Un estudio exploratorio. Anales de la Psicología, 18(2), 273292.

Dergan, J. J. (1999). Hans Jürgen Eysenck: Su contribución a la psicología contemporánea. Revista de la Facultad de Psicología de la Universidad Nacional Mayor de San Marcos, 3(4), 151-158.

Dominguez, S.; Villegas, G.; Yauri, C.; Aravena, S. \& Ramírez, F. (2013). Análisis psicométrico preliminar de la forma corta de EDP-R en una muestra de estudiantes universitarios de Lima Metropolitana. Avances en Psicología, 21(1), 7382.
Echeburúa, E. (1992). Psicopatología, variables de personalidad y vulnerabilidad psicológica al juego patológico. Psicothema, 4(1), 7-20.

Engler, B. (1999). Introducción a las teorías de la personalidad. México: McGraw-Hill.

Escalante, E. \& Agrelo, A. (2012). Explorando experiencias en Facebook: Identidad, vínculos sociales, privacidad. Revista Peruana de Psicología y Trabajo Social, 1(1), 69-88.

Escurra, L. M. y Salas, E. (2014). Construcción y validación del cuestionario de adicción a redes sociales (ARS). Liberabit, 20(1), 73-91.

Estallo, J. A. (1994). Videojuegos, personalidad y conducta. Psicothema, 6(2), 181-190.

Eysenck, J. H. (1982). Las bases biológicas de la personalidad. Barcelona: Fontanella.

Flores, C.; Gamero, K.; Arias, W. L.; Melgar, C.; Sota, A. \& Ceballos, K. (2015). Adicción al celular en estudiantes de la Universidad Nacional de San Agustín y la Universidad Católica San Pablo. Revista de Psicología de la Universidad Católica San Pablo, 5(2), 13-25.

Fuentes, J. B. (2002). Theodore Millon, personólogo y psicopatólogo: La importancia crítica de los trastornos de la personalidad para la psicología. Revista de Historia de la Psicología, 23(3-4), 353-362.

Geldres, J. A. \& Arias, W. L. (2008). Descripción de la personalidad a través de la selección de adjetivos en estudiantes universitarios. Revista de Psicología de la UCSM, 5, 77-85.

Gómez, L. (1985). Dimensiones de personalidad y valores interpersonales en adolescentes. Revista Latinoamericana de Psicología, 17(2), 193-203.

Infante, M. A. (2013). Criptografía y psicología de la contraseña: generando una contraseña fuerte para diferentes servicios. Apuntes en Ciencia $y$ Sociedad, 3(1), 71-75.

Laak, J. (1996). Las cinco grandes dimensiones de la personalidad. Revista de Psicología de la PUCP, 14(2), 129-180.

Lemos, S. (1989). Esquizofrenia: Componentes de la personalidad como factores de riesgo. Psicothema, 1(1), 55-69. 
Machicao, R. (2012). Factores de personalidad y actitud hacia los residuos sólidos en estudiantes de una universidad particular de Lima - Perú. Revista de Psicología Herediana, 7(1-2), 21-30.

Mikusinski, E. B.; Omar, A. G.; Quintana, M. E. L. \& Andrade, M. C. (1980). Exploración de las dimensiones eysenckianas de personalidad mediante el diferencial semántico. Revista Latinoamericana de Psicología, 12(3), 425-440.

Montaña, C.; Acosta, M. A. \& Gerena, R. M. (2002). Estudio descriptivo correlacional entre ira y personalidad a la luz de la teoría de Eysenck. Revista Colombiana de Psicología, 11, 56-70.

Richaud, M.C.(2002). Una revisión crítica del enfoque lexicográfico y del modelo de los Cinco Factores. Revista de Psicología de la PUCP, 20(1), 5-25.

Richaud, M. C. (2003). Nuevos métodos estadísticos para la investigación en evaluación de la personalidad. Revista de Psicología de la PUCP, 21(2), 291-310.

Ruíz, V. M. (2003). El modelo estructural de Cinco Grandes factores de personalidad: medio siglo de historia (1949-1999). Revista de Historia de la Psicología, 24(1), 63-91.

Salas, E. (2014). Adicciones psicológicas y los nuevos problemas de salud. Cultura, 28, 111-146.
Salas, E. y Escurra, L. M. (2014). Uso de redes sociales entre estudiantes universitarios limeños. Revista Peruana de Psicología y Trabajo Social, 3(1), 7590.

Sanabria, P. A. (2004). Características psicológicas de consumidores de cibersexo: Una aproximación. Acta Colombiana de Psicología, 12, 19-38.

Sánchez, M. P.; Thorne, C.; Martínez, P.; Niño de Guzmán, I. \& Argumedo, D. (2002). Adaptación del Inventario de Estilos de Personalidad de Millon en una población universitaria peruana. Revista de Psicología de la PUCP, 20(1), 27-53.

Schmidt, V.; de Costa, M. E.; Firpo, F.; Vion, D. \& Casella, L. (2008). Hans Jürgen Eysenck. Vida y obra de uno de los más influyentes científicos de la historia de la psicología. Avances en Psicología Latinoamericana, 26(2), 304-317.

Vallejo, M. A.; Jordán, C. M.; Díaz, M. I.; Comeche, M. I.; Ortega, J.; Amor, P. J. \& Zubeidat, I. (2008). Perfil sociodemográfico de los usuarios de un servicio de asistencia psicológica on line. Psicothema, 20(4), 610-615.

Zambrano, R. (2011). Revisión sistemática del Cuestionario de Personalidad de Eysenck (EPQ). Liberabit, 17(2), 147-155.

Fecha de recepción: 30 de octubre de 2016

Fecha de aceptación: 15 de noviembre de 2016 